\title{
Identification of Metabolomic Biomarkers for Endometrial Cancer and Its Recurrence after Surgery in Postmenopausal Women
}

\author{
Yannick Audet-Delage ${ }^{1}$, Lyne Villeneuve ${ }^{1}$, Jean Grégoire², Marie Plante² \\ and Chantal Guillemette ${ }^{1,3 *}$ \\ ${ }^{1}$ Centre Hospitalier Universitaire (CHU) de Québec Research Center, Faculty of Pharmacy, Laval University, Québec, QC, \\ Canada, ${ }^{2}$ Gynecologic Oncology Service, CHU de Québec, Department of Obstetrics, Gynecology, and Reproduction, \\ Faculty of Medicine, Laval University, Québec, QC, Canada, ${ }^{3}$ Canada Research Chair in Pharmacogenomi, Laval University, \\ Québec, QC, Canada
}

OPEN ACCESS

Edited by: Haim Werner,

Tel Aviv University, Israel

Reviewed by:

Ann E. Walts,

Cedars-Sinai Medical Center,

United States

Ilan Bruchim,

Hillel Yaffe Medical Center, Israe

*Correspondence:

Chantal Guillemette chantal.guillemette@crchudequebec.

ulaval.ca

Specialty section:

This article was submitted to

Cancer Endocrinology,

a section of the journal

Frontiers in Endocrinology

Received: 20 December 2017

Accepted: 23 February 2018

Published: 12 March 2018

Citation:

Audet-Delage Y, Villeneuve L,

Grégoire J, Plante $M$ and

Guillemette C (2018) Identification of

Metabolomic Biomarkers for

Endometrial Cancer and Its

Recurrence after Surgery in

Postmenopausal Women.

Front. Endocrinol. 9:87.

doi: 10.3389/fendo.2018.00087
Endometrial cancer (EC) is the most frequent gynecological cancer in developed countries. Most EC occurs after menopause and is diagnosed as endometrioid (type I) carcinomas, which exhibit a favorable prognosis. In contrast, non-endometrioid (type II) carcinomas such as serous tumors have a poor prognosis. Our goal was to identify novel blood-based markers associated with EC subtypes and recurrence after surgery in postmenopausal women. Using mass spectrometry-based untargeted metabolomics, we examined preoperative serum metabolites among control women $(n=18)$ and those with non-recurrent (NR) and recurrent $(R)$ cases of type I endometrioid $(n=24)$ and type II serous $(n=12)$ carcinomas. $R$ and NR cases were similar with respect to pathological characteristics, body mass index, and age. A total of 1,592 compounds were analyzed including 14 different lipid classes. When we compared EC cases with controls, 137 metabolites were significantly different. A combination of spermine and isovalerate resulted in an age-adjusted area under the receiver-operating characteristic curve $\left(A \cup C_{a d}\right)$ of $0.914(P<0.001)$ for EC detection. The combination of 2-oleoylglycerol and TAG42:2-FA12:0 allowed the distinction of $R$ cases from NR cases with an $A_{U} C_{\text {adj }}$ of $0.901(P<0.001)$. Type I R cases were also characterized by much lower levels of bile acids and elevated concentrations of phosphorylated fibrinogen cleavage peptide, whereas type II R cases displayed higher levels of ceramides. The findings from our pilot study provide a detailed metabolomics study of EC and identify putative serum biomarkers for defining clinically relevant risk groups.

\section{Keywords: metabolomics, endometrial cancer, blood-based biomarkers, recurrence, mass spectrometry}

\section{INTRODUCTION}

Endometrial cancer (EC) is the sole gynecological neoplasm with a rising incidence and mortality and is currently the most common gynecological cancer in the United States, Canada, and other developed countries (1). Occurring predominantly in postmenopausal women, EC is initially treated

Abbreviations: EC, endometrial cancer; R, recurrent; NR, non-recurrent; MS, mass spectrometry; UPLC-MS/MS, ultraperformance liquid chromatography-tandem mass spectrometry; ROC, receiver operating characteristic; AUC, area under the curve; MAGL, monoacylglycerol lipase; PENAO, 4-( $N$-(S-penicillaminylacetyl)amino) phenylarsonous acid. 
by surgery including total hysterectomy, bilateral salpingooophorectomy, and lymph node evaluation. Despite successful surgery, $10-15 \%$ of tumors recur within 5 years with poor treatment outcomes and low survival rates (2).

Predictive and prognostic factors of EC include histological subtype with high-risk features such as high-tumor grade, stage, and deep myometrial invasion (3). Endometrioid type I carcinomas and especially those of low grade exhibit a favorable prognosis and may be cured by primary surgery (4). Type II carcinomas include non-endometrioid histologies better represented by the prototypical serous carcinoma and account for $\sim 10 \%$ of EC. Type II neoplasms represent higher-grade tumors with a more aggressive clinical course, for which recurrence is more frequent and treatment remains a challenge (5). Candidate diagnostic biomarkers such as CA125 and HE4 have been identified; however, their low sensitivity and/or specificity limit their use in the clinic $(6,7)$. ECs are strongly associated with cumulative estrogen exposure, obesity, and other characteristics of metabolic syndrome (8-12). This does not apply only to type I carcinomas, as type II tumors can also be associated with hormonal, reproductive, and metabolic factors (8). Based on the recognition of the biological and prognostic differences between pathogenetic types of EC and given the poor prognosis for recurrent disease, it is critical to develop novel biomarkers.

Metabolomics is defined as the comprehensive analysis of metabolites in a biological specimen and includes a more focused form of metabolomics that surveys lipids (referred to as lipidomics). This approach is becoming a very powerful tool for biomarker discovery and has proven itself useful in the study of many metabolic diseases including cancer. For instance, discoveries related to oncometabolites have highlighted the possibility of unsuspected cellular pathways whose components could serve as diagnostic or prognostic biomarkers or may be therapeutically targeted for disease treatment (13). Still, very few studies have used metabolomics in the context of EC (Table 1). In contrast, more than 30 global and targeted mass spectrometry (MS)-based metabolomics studies have been conducted for ovarian cancer and have identified dysregulated metabolic pathways that underlie several histological types of carcinoma (14). These discoveries have led to the identification of potential new therapeutic targets and biomarkers that might improve diagnosis and prognostication (15-18).

In the present study, our goal was to identify non-invasive biomarkers of EC cancer and recurrence in postmenopausal women using global metabolomics and lipidomics profiling. We examined preoperative serum metabolites in control women as well as those from women with EC of both histological types; these individuals were from a prospective study of women who underwent hysterectomy and were recruited from a single center. We used validated metabolomics platforms capable of identifying and quantifying multiple biochemical species simultaneously across all major metabolite classes as well as complex lipids including phospholipid, sphingolipid, and neutral lipid classes. In the first series of analyses, we identified putative EC biomarkers by comparing EC cases to control women. Then, we compared type I and type II EC cases to find biomarkers associated with histological type. In the third set of analyses, we compared matched recurrent

TABLE 1 | Summary of previous metabolomics studies of EC.

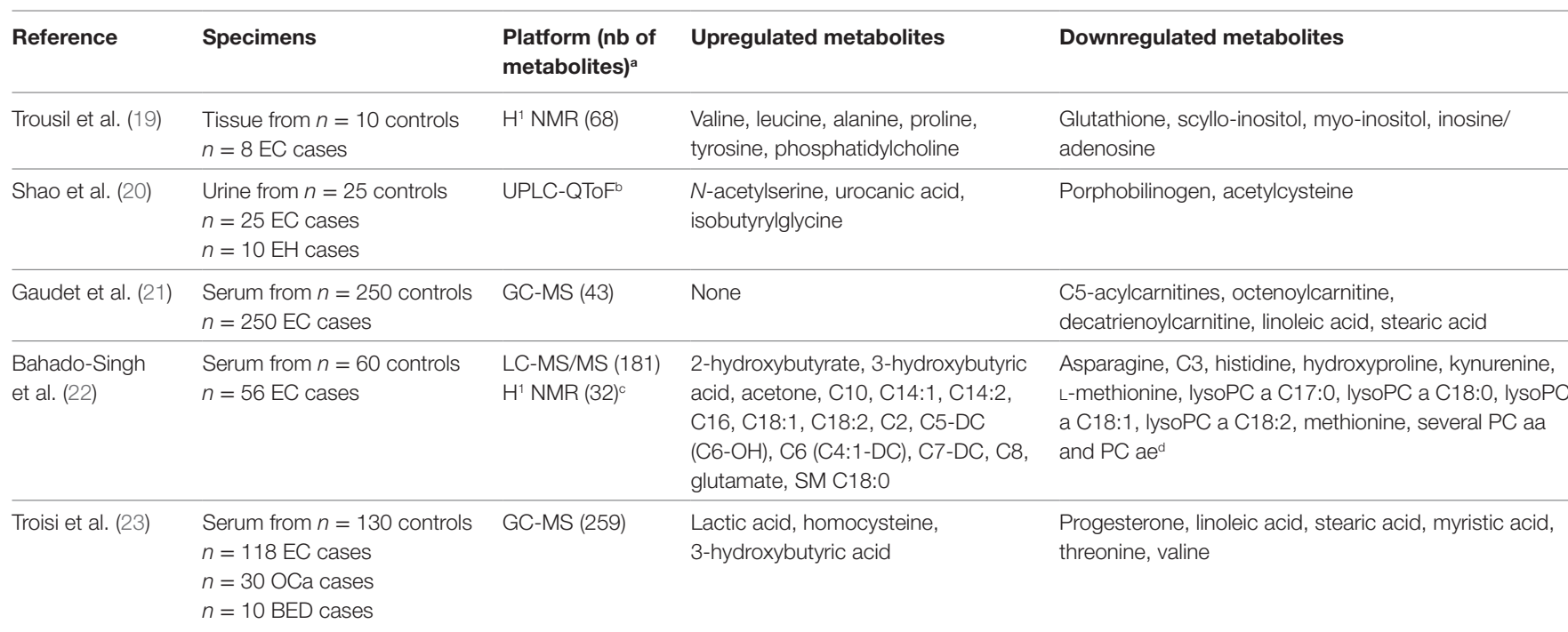

EC, endometrial cancer; EH, endometrial hyperplasia; BED, benign endometrial disease; OCa, ovarian cancer; GC-MS, gas chromatography-mass spectrometry; $H^{\top}$ NMR, proton nuclear magnetic resonance; UPLC-QToF, ultra-performance liquid chromatography-quadrupole time-of-flight mass spectrometry; LC-MS/MS, liquid chromatography-tandem MS; PC, phosphatidylcholines; PC aa, diacyl-phosphatidylcholines; PC ae, acyl-alkyl-phosphatidylcholines.

aThe number of metabolites examined is shown in parentheses.

${ }^{b}$ The authors did not report the number of metabolites detected.

'Metabolites detected by $\mathrm{H}^{\prime}$ NMR were also detected by LC-MS/MS.

${ }^{d} P C$ aa and $P C$ ae were PC aa C36:0, PC aa C36:1, PC aa C36:3, PC aа C36:5, PC aa C36:6, PC aa C38:0, PC aa C38:5, PC aa C40:2, PC aa C42:2, PC aa C42:6, PC ae C34:0, PC ae C34:2, PC ae C34:3, PC ae C36:1, PC ae C36:2, PC ae C36:3, PC ae C38:0, PC ae C38:1, PC ae C38:2, PC ae C38:5, PC ae C38:6, PC ae C40:1, PC ae C40:6, $P C$ ae C42:1, $P C$ ae C42:2, $P C$ ae $C 42: 3$. 
(R) and non-recurrent (NR) cases of both histological type I and type II to detect recurrence biomarkers of EC. Finally, to identify putative biomarkers that would be specific to either type I or type II carcinomas, we compared matched R and NR cases of individual histological type.

\section{MATERIALS AND METHODS}

\section{Study Population}

All participants provided written informed consent for their participation in the study and for the use of their specimens. The current study was evaluated and approved by the local Ethical Research Committee of the Centre Hospitalier Universitaire (CHU) de Québec-Université Laval (2012-993) and was conducted in accordance with the Declaration of Helsinki. The recruitment and specimen collection have been described (8). Briefly, participants were all recruited at the Hôtel-Dieu de Québec Hospital (Québec City, QC, Canada), between 2002 and 2014. All women were of postmenopausal status and underwent surgery (hysterectomy and bilateral salpingo-oophorectomy), either for EC treatment or for non-malignant conditions $(n=9$ for benign pelvic mass, $n=3$ for prophylactic treatment, $n=3$ for precancerous cervical lesion, $n=2$ for fibroma, $n=1$ for uterine prolapse). Fasting blood samples were collected on the morning of surgery and were rapidly processed and stored at $-80^{\circ} \mathrm{C}$ until analysis. To be eligible, women must not have developed prior malignancies nor taken hormone replacement therapy (HRT) during the 3 weeks preceding specimen collection. EC recurrence was ascertained by computerized tomography scan and further confirmed by histopathology when required. Nurses collected information regarding demographic and anthropometric data through standardized questionnaires. A pathologist assessed the histopathological characteristics of the hysterectomy specimens for women with EC. Systematic compilation and review of medical records were performed by one of the treating gynecologic oncologists (Jean Grégoire), for both cases and controls.

Recurrent EC cases consisted of endometrioid $(n=12)$ or serous carcinoma $(n=6)$. To reduce potential confounding factors, NR EC cases were matched to R cases according to (i) histological type, (ii) grade, (iii) a body mass index (BMI) within an interval of $5 \mathrm{~kg} \mathrm{~m}^{-2}$, and (iv) age. In addition, (v) myometrial invasion was also considered for the matching of R and NR type I cases. We achieved a perfect match for the first two criteria for both histological types, as well as myometrial invasion for type I carcinomas. The median difference in BMI was $2.0 \mathrm{~kg} \mathrm{~m}^{-2}$. For two pairs of matched cases, the differences in BMI were of 7.2 and $11.2 \mathrm{~kg} \mathrm{~m}^{-2}$ (Table S1 in Supplementary Material). The median difference in age was 7 years. Control women were not matched to $\mathrm{EC}$ cases.

\section{Metabolomics}

Serum sample aliquots were analyzed for metabolites and lipids with the metabolomics platform at Metabolon Inc. (Durham, NC, USA). Global profiling was conducted as described (24). Briefly, samples were prepared using the automated MicroLab STAR system (Hamilton Company, Reno, NV, USA). A recovery standard was added prior to the first step in the extraction process for quality control purposes. Metabolites were extracted by vigorous agitation after precipitation of proteins with methanol. Samples were then split to enable analysis by different methods, utilizing a Waters ACQUITY ultra-performance liquid chromatography (UPLC) system coupled to a Thermo Scientific Q-Exactive highresolution accurate-mass spectrometer equipped with a heated electrospray ionization source and an Orbitrap mass analyzer. Raw data extraction, peak identification, and quality control processing were carried out using the Metabolon proprietary hardware and software. Compound identification was done through comparison with a library of chromatographic and MS data from authenticated standards.

Complex lipid profiling was conducted according to a modified version of a previously described protocol (25). Briefly, lipids were extracted from serum samples by a heptane/ethyl acetate mixture after addition of a butanol/methanol solution. Phase separation was induced by addition of aqueous acetic acid and centrifugation. MS analysis was conducted on a Shimadzu LC with nano PEEK tubing coupled to a Sciex SelexIon-5500 QTRAP. The scan was performed in multiple reaction monitoring mode. Peaks were quantified using the area under the curve (AUC) method, and data were normalized for inter-day signal differences. The analytical variability was $\leq 10 \%$ for both global profiling and lipidomics.

\section{Statistical Analyses}

The similarity between groups (EC cases vs. controls, R vs. NR cases) was assessed by Student's two-sample $t$-test for continuous variables, with correction for variance unequality when required (Welch's two-sample $t$-test). Chi-square tests were used for categorical data or Fisher's exact test when appropriate. Metabolomics data were log-transformed prior to statistical comparisons, and fold changes (FCs) were calculated based on the geometric mean. The Welch's two-sample $t$-test was conducted for all comparisons, as it offers a slightly better statistical precision than paired sample analysis, which could have been used for the comparison of R to NR cases (26). Pathway enrichment analyses were performed with Metabolon online tools and using their proprietary database. The enrichment score was calculated by dividing the ratio of statistically significant metabolites within a pathway by the overall proportion of statistically significant metabolites.

Predicted probabilities, calculated with logistic regression, were used to build univariate and multivariate receiver operating characteristic (ROC) curves for EC and recurrence detection. Univariate regression models were made for each of the top four altered metabolites, and multivariate models were adjusted for age. BMI did not significantly contribute to the models $(P>0.80)$. Multivariate models were then optimized to give the best AUC with a maximum of four metabolites. The size of our group allowed the detection $(\alpha=0.05,1-\beta=0.80)$ of an AUC $>0.700$ for EC detection, and an AUC $>0.730$ for recurrence (27). Finally, because of the exploratory nature of the study, as well as the number of metabolites tested $(n=1,592)$, statistical adjustment for multiple tests was not performed. 


\section{RESULTS}

\section{Markers of EC - Comparison of all EC Cases Relative to Controls}

We examined preoperative serum metabolites from control women $(n=18)$ and from women with $\mathrm{NR}(n=18)$ or $\mathrm{R}(n=18)$ cases of either type I ( $n=12 \mathrm{R}$ and $n=12 \mathrm{NR}$ ) or type II ( $n=6 \mathrm{R}$ and $n=6 \mathrm{NR}$ ) carcinomas. To reduce biases that may be caused by menstrual cycling, all of the women were postmenopausal, and none of them had used HRT in the 3 weeks preceding specimen collection. All individuals were selected from a larger cohort recruited at a single center (8), and pairing of NR cases with R cases was based on pathological (histological type, grade, and myometrial invasion) and clinical (BMI and age) characteristics (Table 2; Table S1 Supplementary Material). A total of 1,592 compounds of known identity across all major metabolite classes, and 14 different lipid classes were assessed by UPLC-tandem MS using global profiling and lipidomics.

When comparing EC cases to control women, 137 metabolites were significantly altered ( 115 up and 22 down, $P<0.05$; Figure $S 1$ in Supplementary Material). Pathway enrichment analysis identified lipid- and glycolysis-related pathways as the most affected

TABLE 2 | Demographics of control postmenopausal women and those who were newly diagnosed with endometrial cancer (EC).

\begin{tabular}{|c|c|c|c|c|c|c|}
\hline \multirow[b]{2}{*}{ Characteristic } & & & \multicolumn{4}{|c|}{ EC cases $(n=36)$} \\
\hline & \multicolumn{2}{|c|}{$\begin{array}{l}\text { Controls } \\
(n=18)\end{array}$} & \multicolumn{2}{|c|}{$\begin{array}{c}\text { Non-recurrent } \\
(n=18)\end{array}$} & \multicolumn{2}{|c|}{$\begin{array}{c}\text { Recurrent } \\
\quad(n=18)\end{array}$} \\
\hline Continuous variable data & \multicolumn{2}{|c|}{ Mean \pm SD } & \multicolumn{2}{|c|}{ Mean \pm SD } & \multicolumn{2}{|c|}{ Mean \pm SD } \\
\hline Age (years) & \multicolumn{2}{|c|}{$58.9 \pm 10.4^{a}$} & \multicolumn{2}{|c|}{$66.3 \pm 8.3$} & \multicolumn{2}{|c|}{$67.5 \pm 9.4$} \\
\hline Height (cm) & \multicolumn{2}{|c|}{$159.2 \pm 5.3$} & \multicolumn{2}{|c|}{$157.9 \pm 5.4$} & \multicolumn{2}{|c|}{$156.5 \pm 6.7$} \\
\hline Weight (kg) & \multicolumn{2}{|c|}{$70.1 \pm 20.1$} & \multicolumn{2}{|c|}{$70.7 \pm 16.9$} & \multicolumn{2}{|c|}{$68.3 \pm 14.1$} \\
\hline $\mathrm{BMI}$ & \multicolumn{2}{|c|}{$27.5 \pm 7.2$} & \multicolumn{2}{|c|}{$28.4 \pm 7.0$} & \multicolumn{2}{|c|}{$28.0 \pm 6.4$} \\
\hline Mean follow-up (months) & \multicolumn{2}{|c|}{$\mathrm{NA}$} & \multicolumn{2}{|c|}{$56.3 \pm 26.5$} & \multicolumn{2}{|c|}{$65.4 \pm 48.7$} \\
\hline Categorical data & $n$ & (\%) & $n$ & (\%) & $n$ & $(\%)$ \\
\hline \multicolumn{7}{|l|}{ Full-term pregnancy } \\
\hline No & 4 & $(22)$ & 7 & (39) & 8 & (44) \\
\hline Yes & 14 & (78) & 10 & (56) & 9 & (50) \\
\hline Missing & 0 & $(0)$ & 1 & (6) & 1 & (6) \\
\hline \multicolumn{7}{|l|}{ OC use } \\
\hline No & 8 & $(44)$ & 10 & (56) & 12 & $(67)$ \\
\hline Yes & 10 & (56) & 7 & (39) & 5 & (28) \\
\hline Missing & 0 & (0) & 1 & (6) & 1 & (6) \\
\hline \multicolumn{7}{|l|}{ Smoking } \\
\hline Never & 12 & $(67)$ & 11 & (61) & 13 & (72) \\
\hline Current & 4 & (22) & 4 & (22) & 3 & (17) \\
\hline Ex-smoker & 2 & (11) & 3 & (17) & 2 & (11) \\
\hline \multicolumn{7}{|l|}{$\mathrm{HRT}$} \\
\hline No & 14 & (78) & 10 & $(56)$ & 11 & (61) \\
\hline Yes & 4 & (22) & 7 & (39) & 6 & (33) \\
\hline Missing & 0 & $(0)$ & 1 & (6) & 1 & (6) \\
\hline
\end{tabular}

${ }^{a}$ Control women were slightly younger than $E C$ cases $(P<0.05)$. No other significant differences were noted between cases and controls. No statistical differences were noted between $R$ and NR cases. Student's t-test was used for continuous variable data, with adjustment for variance inequality when required (Welch's two-sample t-test). Categorical data were assessed using the chi-square test $\left(\chi^{2}\right)$ or Fisher's exact test, when applicable.

HRT, hormone replacement therapy; OC, oral contraceptive; BMI, body mass index; NA, not applicable. in EC cases (Figure 1A). Conjugated forms of lipids, such as acylcholines, monoacylglycerols, and acylcarnitines, were generally higher in EC cases as compared with control women, whereas free fatty acids were detected at lower concentrations, supporting a remodeling of fatty acid metabolism in EC (Figure 1B). Of note, the $\mathrm{C} 5$ acylcarnitine 2-methylbutyrylcarnitine was also elevated in $\mathrm{EC}$ cases $(\mathrm{FC}=1.27, P=0.023)$. Five of the top 10 most altered features in EC cases were peptides and amino acids (Table 3), with spermine ( $\mathrm{FC}=7.66, P=0.0004)$, and isovalerate $(\mathrm{FC}=-2.56$, $P=0.015)$ as the most changed metabolites in cancer cases.

To further assess our ability to distinguish EC cases from controls based on these metabolites, we constructed ROC curves based on univariate and multivariate logistic regression models. The combination of spermine and isovalerate resulted in an area under the ROC curve (AUC) of 0.875 [95\% confidence interval $(\mathrm{CI})=0.784-0.966]$, and an age-adjusted AUC $\left(\mathrm{AUC}_{\mathrm{adj}}\right)$ of $0.914(95 \% \mathrm{CI}=0.833-0.994)$, very similar to a more complete model that included spermine, isovalerate, glycylvaline, and gamma-glutamyl-2-aminobutyrate and resulted in an $\mathrm{AUC}_{\text {adj }}$ of 0.921 (95\% CI $=0.843-1.000)$ (Figure 1C). These results support the capacity of these metabolites to discriminate EC cases from controls.

\section{Markers Associated with Histological Types - Comparison of Type I and Type II EC Cases}

A total of 98 metabolites significantly distinguished type I from type II ECs ( $n=30$ higher in type I, $n=68$ lower in type I, $P<0.05)$. The most different metabolites between histotypes were bradykinin, with higher levels in type $\mathrm{I}(\mathrm{FC}=2.70, P=0.003)$, and heme, which was 4.52 -fold higher in type II ECs $(P=0.030)$ (Table 3). Levels of saturated long-chain acylcarnitines were higher in type II, with C20, C24, and C26 acylcarnitines displaying FCs of $1.32(P=0.021), 1.33(P=0.027)$, and $1.38(P=0.005)$, respectively.

Levels of choline were higher $(\mathrm{FC}=1.27, P=0.010)$ in type II ECs, along with sarcosine $(\mathrm{FC}=1.42, P=0.023)$, which are both metabolites of the tetrahydrofolate-serine/glycine pathway. Glycine levels tended to be elevated in type II ECs as well (FC $=1.23, P=0.075)$. Levels of sulfated androgenic steroids differed significantly between the two histotypes, with type I EC having higher levels than type II for 10 out of the 18 androgenic compounds assessed by the method.

\section{Markers of EC Recurrence - Comparison of R and NR Cases of Both Histological Types}

Recurrent cases were characterized by an altered lipid metabolism relative to NR cases. Among the 104 metabolites modulated, 80 represented lipid metabolism (68/75 up and 12/29 down; $P<0.05$ ). Pathway enrichment analysis (Figure 2A) identified many classes of lipids affected in R cases, such as monoacylglycerols, for which 16:1, 18:1, 20:5, and 22:6 species of both alpha and beta isomers were significantly elevated (Figure 2B; Table 4).

In addition to modifications in lipid levels, other classes of compounds displayed significant alterations in $\mathrm{R}$ cases when 


\section{A Top enriched pathways in EC cases $\quad$ C ROC curves}

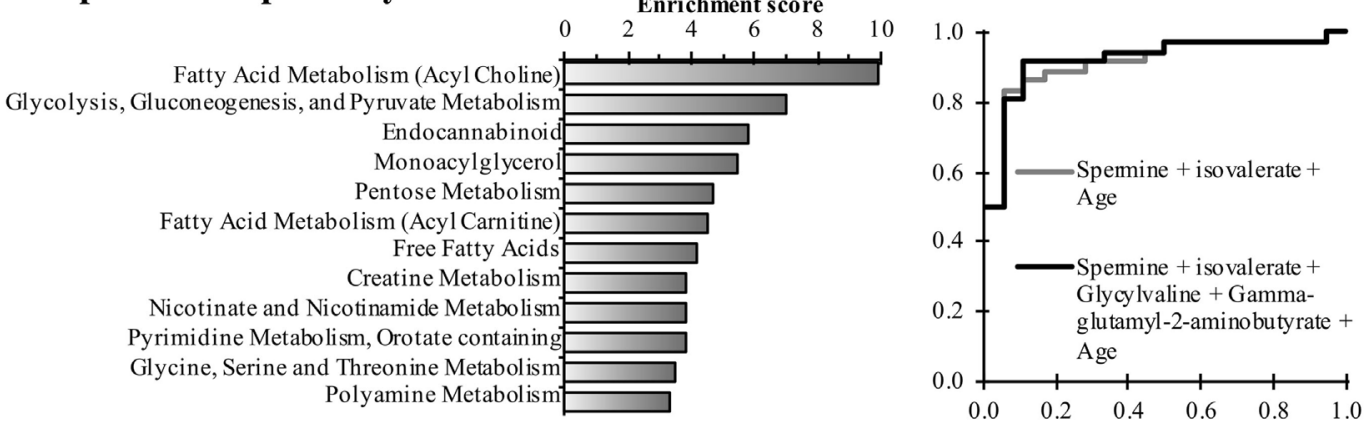

\section{B Lipid remodeling in $\mathrm{EC}$ cases}

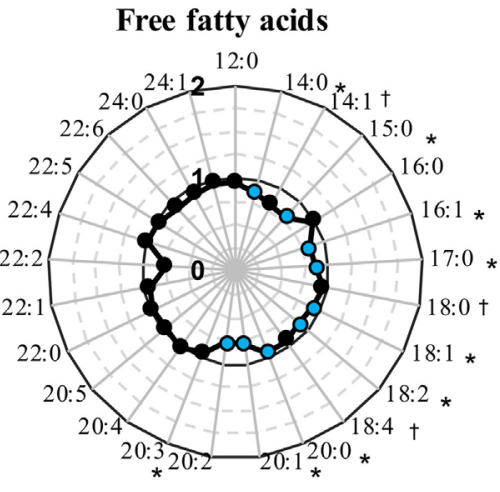

Monoacylglycerols

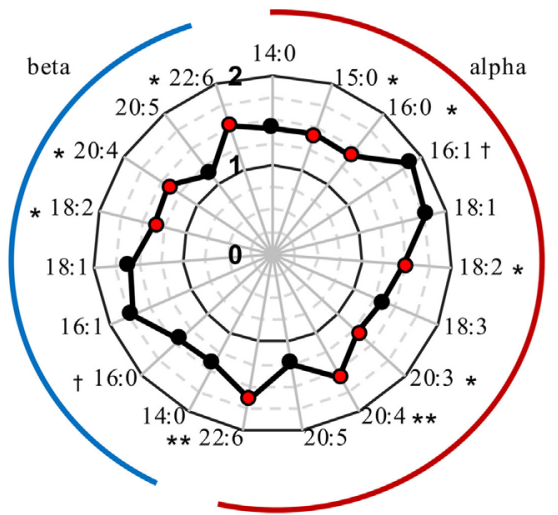

Acylcarnitines

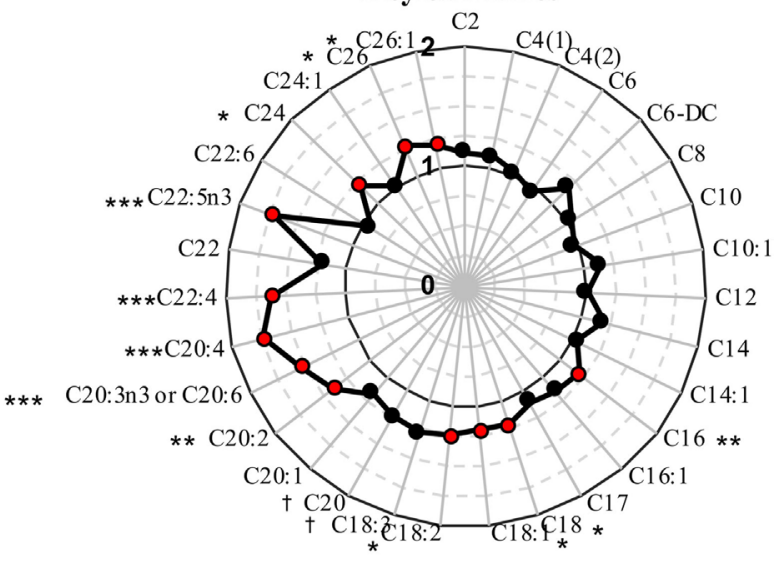

Acylcholines

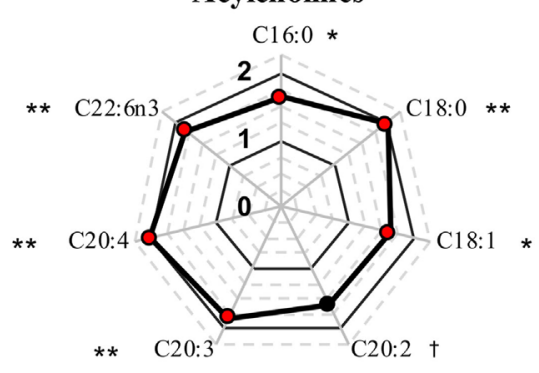

FIGURE 1 | Comparison of endometrial cancer (EC) cases and controls reveals that lipid metabolism is perturbed in EC cases. (A) Pathway enrichment analysis is based on enrichment scores. Pathways containing at least three metabolites and having an enrichment score $>3$ are displayed. (B) Free fatty acid levels are lower in EC cases, whereas conjugated forms of fatty acids are elevated. Fold changes are displayed in radar graphs. Significantly enriched and depleted metabolites are marked in red and blue circles, respectively. (C) Receiver operating characteristic (ROC) curves of the most accurate regression models for detecting EC. ${ }^{\dagger} P<0.10$, ${ }^{\star} P<0.05,{ }^{\star *} P<0.01$.

compared with NR cases. For example, the pathway of glycine, serine, and threonine metabolism was affected, as both serine and threonine levels were lower in $\mathrm{R}$ cases, whereas the glycine precursor $\mathrm{N}$-acetylglycine was elevated (Figure 2C). Even though higher levels of $\mathrm{N}$-acetylglycine were observed, glycine was not affected, suggesting a rerouting of glycine metabolism intermediates in $\mathrm{R}$ cases.

Some metabolites had a similar association with recurrence in both type I and type II EC patients. This was the case of the monoacylglycerol 1-oleoylglycerol (18:1), the only metabolite observed among the top modulated metabolites for both histological types (Table 4), which displayed a FC of $3.77(P=0.045)$ and $2.29(P=0.018)$ for type I and type II, respectively. A similar observation was noted for other lipid metabolites, namely the acylcarnitine docosahexaenoyl carnitine $(\mathrm{C} 22: 6)(\mathrm{FC}=1.51$ and 1.46 for type I and type II, respectively; $P<0.05)$ and the monohydroxylated fatty acids 2-hydroxypalmitate (2-OHC16:0, FC $=1.47$ and 1.57 for type I and type II; $P<0.05)$ and 
TABLE 3 | Top 10 modulated preoperative serum metabolites in endometrial cancer (EC) cases.

\begin{tabular}{|c|c|c|c|}
\hline Subpathway & Biochemical name & $\begin{array}{l}\text { Fold } \\
\text { change }\end{array}$ & $\boldsymbol{P}$-value \\
\hline \multicolumn{4}{|c|}{ Biomarkers of EC } \\
\hline \multicolumn{4}{|c|}{ EC cases $(n=36)$ vs. control postmenopausal women $(n=18)$} \\
\hline $\begin{array}{l}\text { Leucine, isoleucine, and valine } \\
\text { metabolism }\end{array}$ & Isovalerate & -2.56 & 0.0154 \\
\hline Gamma-glutamyl amino acid & $\begin{array}{l}\text { Gamma-glutamyl-2- } \\
\text { aminobutyrate }\end{array}$ & -1.72 & 0.0170 \\
\hline Fatty acid, dicarboxylate & Adipate & -1.64 & 0.0456 \\
\hline $\begin{array}{l}\text { Nicotinate and nicotinamide } \\
\text { metabolism }\end{array}$ & 1-methylnicotinamide & -1.47 & 0.0118 \\
\hline Histidine metabolism & Trans-urocanate & -1.45 & 0.0125 \\
\hline $\begin{array}{l}\text { Methionine, cysteine, SAM, and } \\
\text { taurine metabolism }\end{array}$ & Cystathionine & 2.73 & 0.0011 \\
\hline Secondary bile acid metabolism & Isoursodeoxycholate & 3.40 & 0.0146 \\
\hline Glycogen metabolism & Maltose & 3.41 & 0.0005 \\
\hline Dipeptide & Glycylvaline & 3.92 & 0.0075 \\
\hline Polyamine metabolism & Spermine & 7.66 & 0.0004 \\
\hline \multicolumn{4}{|c|}{ Biomarkers of EC histological types } \\
\hline \multicolumn{4}{|c|}{ Type II EC cases $(n=12)$ vs. type I EC cases $(n=24)$} \\
\hline Polypeptide & Bradykinin, des-arg(9) & -2.70 & 0.003 \\
\hline Androgenic steroids & $\begin{array}{l}\text { Androsteroid monosulfate } \\
\mathrm{C} 19 \mathrm{H} 2806 \mathrm{~S}\end{array}$ & -2.33 & 0.030 \\
\hline Xanthine metabolism & 1,3,7-trimethylurate & -2.33 & 0.047 \\
\hline Androgenic steroids & $\begin{array}{l}\text { 5-alpha-androstan-3beta, } \\
\text { 17beta-diol disulfate }\end{array}$ & -2.17 & 0.025 \\
\hline Androgenic steroids & $\begin{array}{l}\text { Androstenediol (3alpha, } \\
\text { 17alpha) monosulfate }\end{array}$ & -2.08 & 0.017 \\
\hline TAG ester & TAG42:1-FA12:0 & 2.92 & 0.049 \\
\hline TAG ester & TAG46:3-FA18:3 & 3.05 & 0.030 \\
\hline TAG ester & TAG44:2-FA12:0 & 3.26 & 0.038 \\
\hline TAG ester & TAG44:2-FA18:2 & 3.38 & 0.041 \\
\hline $\begin{array}{l}\text { Hemoglobin and porphyrin } \\
\text { metabolism }\end{array}$ & heme & 4.52 & 0.030 \\
\hline
\end{tabular}

2-hydroxystearate (2-OH-C18:0, FC $=1.30$ and 1.56 for type I and type II; $P<0.05$ ), suggesting a remodeling of lipid metabolism among $\mathrm{R}$ cases of both histological types when compared with NR cases. In addition, these four metabolites were not significantly different when comparing EC cases and control women (data not shown), suggesting their relevance as biomarkers of recurrence, independently of the histological type.

Receiver operating characteristic curves identified 2-oleoylglycerol and TAG42:2-FA12:0 as the most effective metabolites to discriminate $\mathrm{R}$ cases from NR cases, with an AUC $=0.877(95 \%$ $\mathrm{CI}=0.730-0.990)$ and an $\mathrm{AUC}_{\text {adj }}=0.901$ (95\% CI $\left.=0.796-1.000\right)$. These results are similar to the model including more metabolites, namely 1-oleoylglycerol, 2-oleoylglycerol, TAG40:0-FA12:0, and TAG42:2-FA12:0, which displayed an $\mathrm{AUC}_{\text {adj }}=0.904(95 \%$ $\mathrm{CI}=0.807-1.000$; Figure 2D), confirming the ability of these metabolites to predict recurrence.

\section{Markers Associated with Recurrence According to Histological Types - Comparison of R and NR Cases According to Histological Type}

Several metabolites were specifically associated with recurrence in type I endometrioid or type II serous cases. For instance, modifications in bile acid metabolism were mainly observed in type I R cases, which had lower levels of primary and secondary bile acid metabolites such as taurodeoxycholate $(\mathrm{FC}=-7.14$, $P=0.009)$, glycodeoxycholate $(\mathrm{FC}=-4.55, P=0.009)$, and taurocholate ( $\mathrm{FC}=-3.85, P=0.038$; Figure 3A; Table 4). Type I recurrent cases were also characterized by an enrichment in circulating levels of phosphorylated fibrinogen cleavage peptide ADpSGEGDFXAEGGGVR $(\mathrm{FC}=1.68, P=0.014$; Figure 3B; Table 4); an association not found for type II R cases $(P=0.477)$.

In contrast, multiple classes of sphingolipids were significantly enriched in type II R cases, including ceramides, their precursors dihydroceramides, and their glycosylated derivatives lactosylceramides. Though the variations were modest with FCs between 1.29 and 1.60 for significant metabolites, numerous metabolites of this pathway were similarly altered, underscoring the potential significance of these variations (Figure 3C). None of the ceramides were significantly different in type I R cases, suggesting that alterations in these pathways could be specific to type II R cases.

\section{DISCUSSION}

In this study, we profiled 1,592 compounds in 54 postmenopausal women. To the best of our knowledge, this is the first study reporting metabolites associated with type I and type II EC carcinomas and their recurrence following initial surgical treatment. Our findings represent an important pilot study in the identification of putative serum biomarkers useful for detecting EC and predicting recurrence following initial surgery, to ultimately improve patient survival based on better stratification and informed treatment decisions.

We found that the levels of free fatty acids linoleic acid (C18:2) and myristic acid (C14:0) were lower in EC cases as compared with control women, consistent with previous reports comparing EC cases and controls $(21,23)$. Gaudet et al. (21) also observed modifications in intermediates from the branched chain amino acid pathway, such as isovalerylcarnitine/2-methylbutyrylcarnitine (undistinguishable by the MS method), which were also altered in our set of EC cases compared with controls. Other comparisons between the two studies could not be extended, as their panel of metabolites was targeted to 69 compounds. In our dataset, additional metabolites related to amino acids were affected in EC cases, such as polyamines, which are involved in cancer progression, including endocrine-related neoplasms like breast cancer (28). Accordingly, the most elevated metabolite between EC cases and controls was spermine, a biomarker of EC possibly originating from EC cells. This is conceivable as polyamine synthesis and degradation are actively regulated in the endometrium, notably during the menstrual cycle and pregnancy (29-31).

Lipids were also considerably affected in EC cases when compared with controls, with lower serum concentrations of free fatty acids but higher levels of conjugated fatty acids such as acylcholines, acylcarnitines, and monoacylglycerols. This is consistent with a report from Bahado-Singh et al. (22). They observed higher acylcholine levels in EC patients. However, very little is known about these lipids (no entries were found in either the Human Metabolome Database or Kyoto Encyclopedia 


\section{A Top enriched pathways in $R$ cases

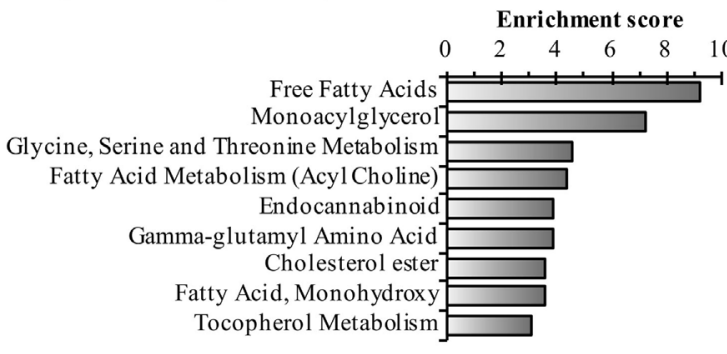

C Glycine, Serine and Threonine Metabolism

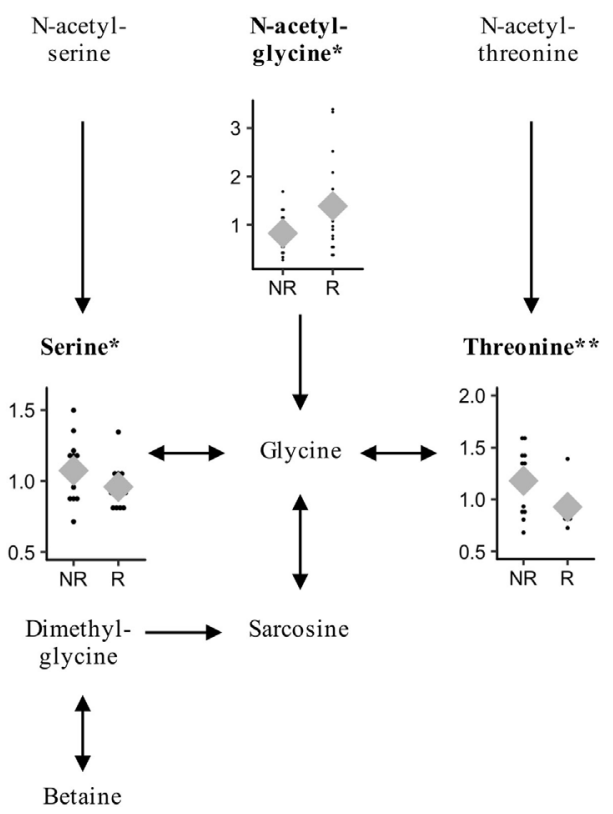

\section{B Monoacylglycerols}

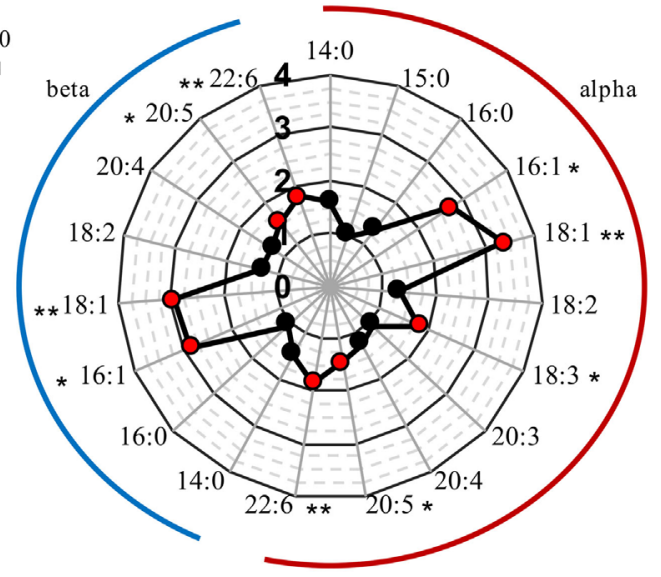

D ROC curves

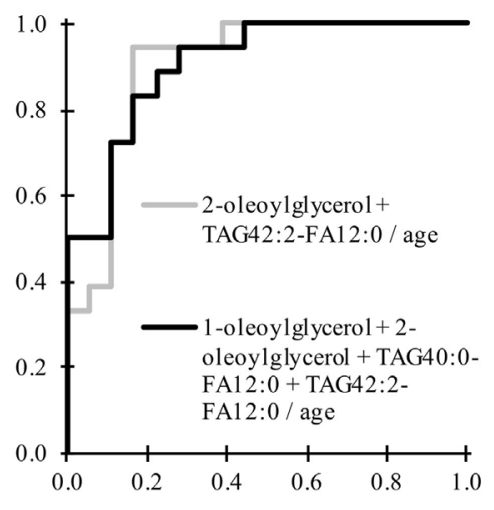

FIGURE 2 | Monoacylglycerols and amino acids are remodeled in Recurrent (R) endometrial cancer cases when compared to Non-Recurrent (NR) cases. (A) Most-enriched pathways in R cases as compared with NR cases. Pathways containing at least three metabolites and having an enrichment score $>3$ are displayed. (B) Several species of monoacylglycerol are elevated in R cases. (C) The metabolism of glycine, serine, and threonine is perturbed in $\mathrm{R}$ cases in comparison to NR cases. Normalized levels of detected metabolites are displayed in dot plots, and means are represented by gray diamonds ( $\diamond)$. (D) Receiveroperating characteristics $(\mathrm{ROC})$ curves of the most-accurate regression models to detect recurrence. ${ }^{\star} P<0.05,{ }^{* \star} P<0.01$.

of Genes and Genomes database; accessed on December 15, 2017), although some acylcholines, including palmitoylcholine, stearoylcholine, and oleoylcholine, enhance estradiol penetration through tissues (32). This could be important in the context of a hormone-sensitive cancer, potentially favoring the estrogenic activity of estradiol in the tumor. In addition, acylcarnitines have been linked to EC, as well as to breast cancer, where they are enriched in hypoxic tissues $(21,33)$. Acylcarnitines are synthesized by cells to fuel mitochondrial fatty acid oxidation. However, the mechanisms by which they are found in circulation remains unclear, although their levels should reflect cellular activity and concentration $(34,35)$. It is thus possible that circulating levels of acylcarnitines could reflect the hypoxic status of tumor cells.

We also observed an accumulation of monoacylglycerols to the detriment of free fatty acids. Monoacylglycerols are mainly derived from enzymatic hydrolysis of triacylglycerols and diacylglycerols and can be further metabolized to free fatty acids through the action of monoacylglycerol lipase (MAGL), an enzyme previously identified to be downregulated in EC (36). Accordingly, a lower MAGL activity could explain, at least in part, the observed accumulation of monoacylglycerols in sera of EC cases as compared with controls. Of note, the monoacylglycerol 1-oleoylglycerol (18:1) was strongly elevated in R cases of both tumor types and could represent a marker of EC recurrence. This is consistent with a role for MAGL in various aspects of tumorigenesis (37).

Modifications in lipid levels could also be related to bradykinin, a putative biomarker of type I EC that is known to activate phospholipase D in EC (38). As an inflammatory mediator, bradykinin triggers kinin-activated pathways. These have been associated with EC and breast cancer progression, supporting the role of bradykinin in tumors originating from steroid 
TABLE 4 | Top 10 modulated preoperative serum metabolites in recurrent endometrial cancer cases.

\begin{tabular}{|c|c|c|c|}
\hline Subpathway & $\begin{array}{l}\text { Biochemical } \\
\text { name }\end{array}$ & $\begin{array}{l}\text { Fold } \\
\text { change }\end{array}$ & P-value \\
\hline \multicolumn{4}{|c|}{ Biomarkers of recurrence after initial surgery } \\
\hline \multicolumn{4}{|c|}{ R cases $(n=18)$ vs. NR cases $(n=18)$ for type I and type II carcinomas } \\
\hline Ester & TAG40:0-FA12:0 & -7.14 & 0.0427 \\
\hline Ester & TAG42:2-FA12:0 & -5.00 & 0.0460 \\
\hline Primary bile acid metabolism & Chenodeoxycholate & -2.86 & 0.0263 \\
\hline Glycogen metabolism & Maltose & -2.78 & 0.0028 \\
\hline Secondary bile acid metabolism & Glycoursodeoxycholate & -2.00 & 0.0343 \\
\hline $\begin{array}{l}\text { Fatty acid metabolism (acyl } \\
\text { glycine) }\end{array}$ & Hexanoylglycine & 2.04 & 0.0454 \\
\hline Monoacylglycerol & $\begin{array}{l}\text { 1-palmitoleoylglycerol } \\
(16: 1)\end{array}$ & 2.73 & 0.0484 \\
\hline Monoacylglycerol & $\begin{array}{l}\text { 2-palmitoleoylglycerol } \\
(16: 1)\end{array}$ & 2.86 & 0.0283 \\
\hline Monoacylglycerol & 2-oleoylglycerol (18:1) & & 0.0076 \\
\hline Monoacylglycerol & 1-oleoylglycerol (18:1) & 3.37 & 0.0046 \\
\hline \multicolumn{4}{|c|}{ Biomarkers of recurrence after initial surgery by histological type } \\
\hline \multicolumn{4}{|c|}{$\begin{array}{c}\text { R cases }(n=12) \text { vs. NR cases }(n=12) \text { for type I endometrioid } \\
\text { carcinomas }\end{array}$} \\
\hline Secondary bile acid metabolism & Taurodeoxycholate & -7.14 & 0.0093 \\
\hline Secondary bile acid metabolism & Glycodeoxycholate & -4.55 & 0.0088 \\
\hline Primary bile acid metabolism & Taurocholate & -3.85 & 0.0383 \\
\hline Glycogen metabolism & Maltose & -3.45 & 0.0065 \\
\hline Primary bile acid metabolism & Glycocholate & -3.23 & 0.0263 \\
\hline Free fatty acids & $\mathrm{FFA}(22: 5)$ & 1.66 & 0.0006 \\
\hline Fibrinogen cleavage peptide & ADpSGEGDFXAEGGGVR & 1.68 & 0.0135 \\
\hline Oxidative phosphorylation & Phosphate & 1.76 & 0.0250 \\
\hline Ester & TAG58:10-FA20:5 & 1.88 & 0.0032 \\
\hline Monoacylglycerol & 1-oleoylglycerol (18:1) & 3.77 & 0.0450 \\
\hline \multicolumn{4}{|c|}{ R cases $(n=6)$ vs. NR cases $(n=6)$ for type II serous carcinomas } \\
\hline $\begin{array}{l}\text { Fatty acid metabolism (Acyl } \\
\text { Carnitine) }\end{array}$ & 3-hydroxybutyrylcarnitine & -2.17 & 0.0494 \\
\hline Pentose metabolism & Ribitol & -1.56 & 0.0192 \\
\hline $\begin{array}{l}\text { Purine metabolism (Hypo) } \\
\text { xanthine/inosine containing }\end{array}$ & Allantoin & -1.45 & 0.0481 \\
\hline Histidine metabolism & Histidine & -1.45 & 0.0028 \\
\hline Glutathione metabolism & 2-aminobutyrate & -1.39 & 0.0132 \\
\hline $\begin{array}{l}\text { Fatty acid metabolism (acyl } \\
\text { choline) }\end{array}$ & docosahexaenoylcholine & 2.18 & 0.0413 \\
\hline Monoacylglycerol & $\begin{array}{l}\text { 1-docosahexaenoylglycerol } \\
(22: 6)\end{array}$ & 2.28 & 0.0036 \\
\hline Monoacylglycerol & 1-oleoylglycerol (18:1) & 2.29 & 0.0175 \\
\hline Phospholipid metabolism & Glycerophosphoinositol & 2.38 & 0.0022 \\
\hline Monoacylglycerol & $\begin{array}{l}\text { 2-docosahexaenoylglycerol } \\
(22: 6)\end{array}$ & 2.55 & 0.0067 \\
\hline
\end{tabular}

sensitive tissues $(39,40)$. Sulfated androgens were also higher in type I EC cases, consistent with the reported implication of sulfated steroids in this histotype $(8,41-44)$. Furthermore, our data identified heme as a putative biomarker of type II EC and highlighted modifications in pathways closely related to heme synthesis, namely the tetrahydrofolate-serine/glycine pathway (45). Heme consumption might participate in endometrial carcinogenesis, being associated with a moderate increase in EC risk (46). Targeting this pathway is currently being tested for the treatment of ovarian cancer and other solid tumors using a new drug, 4-(N-(S-penicillaminylacetyl)amino) phenylarsonous acid (PENAO), acting through the induction of heme degradation by heme oxygenase- $1(47,48)$.
Putative biomarkers of EC recurrence were also identified. Compared with patients with non-recurrent type I carcinomas, those who experienced recurrence after surgery presented alterations in bile acid levels. Bile acids contribute to cholesterol homeostasis, a precursor of the steroids that drive the development and progression of this histological type of EC (10). Recently, we showed that higher levels of circulating steroids are linked to an increased risk of recurrence (43). Numerous enzymatic pathways are involved in the conversion of both bile acids and steroids, including reduction by aldo-keto reductases (49), conjugation by uridine diphospho-glucuronosyltransferases (50), sulfotransferases (51), and sulfatase (52). The reduced levels of bile acids may reflect an altered activity of some of these metabolic pathways in $\mathrm{R}$ type I cases, consistent with previous findings $(8,42)$. Bile acids might also act synergistically with steroids by stimulating EC cell growth, as they enhance myometrium sensitivity to hormones such as oxytocin (53). Finally, bile acids might initiate signaling events, as some of them display inflammatory functions $(54,55)$. In accordance with modifications in the inflammatory status of R type I cases, inflammatory response markers such as the phosphorylated cleavage peptide of fibrinogen were elevated in these patients. This is reinforced by studies that have linked the overexpression of procoagulants with gynecologic malignancies including EC, and further associated this overexpression with more aggressive tumor types (56-60). Although fibrinogen may confer a potential advantage to cancer cells in terms of aggressiveness and dissemination, the underlying mechanisms remain unclear.

For the type II ECs analyzed here, all of which were serous carcinomas, our observations revealed enhanced concentrations of numerous ceramides in preoperative sera of $\mathrm{R}$ cases compared with NR cases. Others have linked alterations of sphingolipids and ceramides in EC with the differentiation status of the tumors, but they did not include type II carcinomas (61-64). Tanaka et al. (65) showed that serous ovarian cancers exhibit elevated levels of glycosylated ceramides, consistent with high expression of galactosyltransferase in tumors. As ovarian serous cancers share similarities with type II serous EC, this raises the possibility that alterations in ceramide metabolism may be common to both tissues $(66,67)$. These bioactive lipids participate in tumor progression and the metastasis process (68) and, therefore, may represent promising biomarkers for non-invasive detection of recurrent type II EC. However, their metabolism in endometrial malignant tumors has been poorly characterized and our investigation is the first to present complex data on ceramide metabolism in the context of EC. Additional studies are thus warranted.

We identified putative cancer-specific and recurrence biomarkers using an unbiased metabolomics approach for type I and type II EC in postmenopausal women. Although exploratory, our study has several strengths including the analysis of postmenopausal cases and controls, as well as R and NR cases of two of the most common histological EC subtypes, in addition to the quantification of an extensive panel of metabolites through a validated metabolomics platform. This approach is powerful for screening a large and diverse set of metabolites but is limited in terms of absolute quantification. Additional limitations to our study include a restricted number of prospective EC cases, 


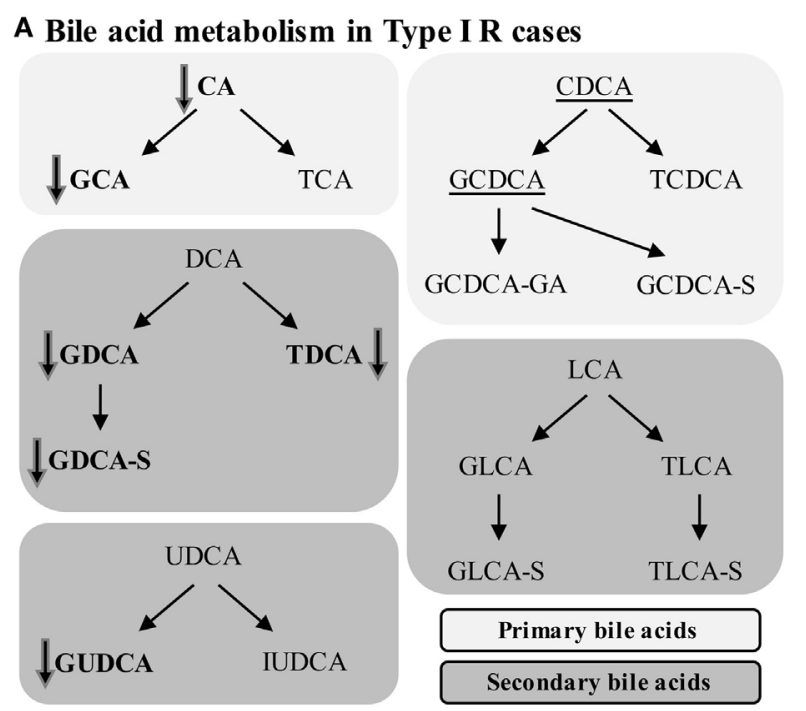

\section{B Phosphorylated fibrinogen peptide in Type I R cases}

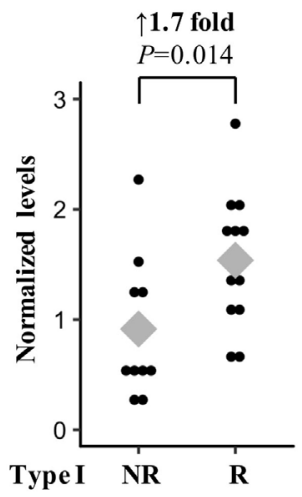

C Ceramides in Type II R cases

\begin{tabular}{|c|c|c|c|c|}
\hline -FA & Dihydroceramide & Ceramide & Hexosylceramide & Lactosylceramide \\
\hline 14:0 & 1.02 & 1.11 & 1.15 & 1.25 \\
\hline $16: 0$ & 1.18 & 1.34 & 1.27 & 1.21 \\
\hline 18:0 & 1.35 & 1.60 & 1.12 & 1.24 \\
\hline $18: 1$ & 1.26 & 1.60 & 1.45 & 1.16 \\
\hline 20:0 & 1.18 & 1.55 & 1.33 & 1.32 \\
\hline $20: 1$ & 1.10 & 1.45 & 1.24 & 1.29 \\
\hline 22:0 & 1.23 & 1.32 & 1.20 & 1.22 \\
\hline 22:1 & 1.17 & 1.40 & 1.31 & 1.24 \\
\hline 24:0 & 1.13 & 1.19 & 1.18 & 1.17 \\
\hline $24: 1$ & 1.18 & 1.28 & 1.12 & 1.18 \\
\hline 26:0 & 1.13 & 1.26 & 1.43 & 1.11 \\
\hline $26: 1$ & 1.17 & 1.19 & 1.09 & 1.29 \\
\hline Total & 1.17 & 1.25 & 1.20 & 1.21 \\
\hline
\end{tabular}

FIGURE 3 | Metabolic alterations of Recurrent (R) endometrial cancer differ between the histological subtypes. (A) R cases of type I histology are associated with reduced bile acid levels. Metabolites in bold were significantly altered $(P<0.05)$, whereas a trend $(P<0.10)$ was detected for underscored metabolites. Bile acids can be conjugated with glucose (G-), taurine (T-), glucuronic acid (-GA), or sulfate (-S). CA, cholic acid; DCA, deoxycholic acid; UDCA, ursodeoxycholic acid; IUDCA, isoursodeoxycholic acid; CDCA, chenodeoxycholic acid; LCA, lithocholic acid. (B) Normalized levels of the phosphorylated fibrinogen cleavage peptide ADpSGEGDFXAEGGGVR were higher in type I R cases. (C) Ceramide levels were significantly altered in type II R cases. Fold change is shown, and significant metabolites $(P<0.05)$ are identified by a black background, whereas a trend $(P<0.10)$ in metabolite differences is shown by gray shading. Cer, ceramide; -FA, fatty acid group; DEGS, dihydroceramide desaturase; CEGT, ceramide glucosyltransferase; GALT, galactosyltransferase; Gal, galactose; Glu, glucose.

whereas the study design likely reduced variations through sample matching. Even though enrolled women must have not taken HRT during the 3 weeks prior to blood draw, it is not known if this period is sufficient to fully restore circulating metabolite levels potentially affected by HRT. Nonetheless, HRT use was similar between groups, which likely reduced the potential bias it might have introduced. The putative biomarkers identified in this pilot study will require validation in larger cohorts using quantitative methods. Their specificity to EC must also be confirmed, notably in comparison to other gynecological malignancies (ovarian cancer, mixed Müllerian cancer) and benign conditions (hyperplasia, endometriosis, etc.), which will facilitate their translation to the clinic. Finally, mechanistic studies are needed to help gain insights into the underlying biological processes driving the observed changes in metabolites in EC cases and those experiencing recurrence after surgery for curative intent. 


\section{ETHICS STATEMENT}

The study was evaluated and approved by local Ethical Research Committees of the Centre Hospitalier Universitaire (CHU) de Québec-Université Laval (2012-993). All subjects gave written informed consent in accordance with the Declaration of Helsinki.

\section{AUTHOR CONTRIBUTIONS}

CG designed and supervised the research. JG and MP were involved in patient recruitment. JG established the clinical database. LV prepared biospecimens. YA-D performed statistical analysis. CG and YA-D took part in the analysis and interpretation of the data and wrote the manuscript. All authors critically reviewed and approved the final version of the manuscript.

\section{REFERENCES}

1. Westin SN, Broaddus RR. Personalized therapy in endometrial cancer: challenges and opportunities. Cancer Biol Ther (2012) 13:1-13. doi:10.4161/ cbt.13.1.18438

2. Buhtoiarova TN, Brenner CA, Singh M. Endometrial carcinoma: role of current and emerging biomarkers in resolving persistent clinical dilemmas. Am J Clin Pathol (2016) 145:8-21. doi:10.1093/ajcp/aqv014

3. Sorbe B. Predictive and prognostic factors in definition of risk groups in endometrial carcinoma. ISRN Obstet Gynecol (2012) 2012:325790. doi: $10.5402 / 2012 / 325790$

4. Han KH, Kim HS, Lee M, Chung HH, Song YS. Prognostic factors for tumor recurrence in endometrioid endometrial cancer stages IA and IB. Medicine (Baltimore) (2017) 96:e6976. doi:10.1097/MD.0000000000006976

5. Mang C, Birkenmaier A, Cathomas G, Humburg J. Endometrioid endometrial adenocarcinoma: an increase of G3 cancers? Arch Gynecol Obstet (2017) 295:1435-40. doi:10.1007/s00404-017-4370-4

6. Rizner TL. Discovery of biomarkers for endometrial cancer: current status and prospects. Expert Rev Mol Diagn (2016) 16:1315-36. doi:10.1080/14737 159.2016.1258302

7. Knific T, Osredkar J, Smrkolj S, Tonin I, Vouk K, Blejec A, et al. Novel algorithm including CA-125, HE4 and body mass index in the diagnosis of endometrial cancer. Gynecol Oncol (2017) 147:126-32. doi:10.1016/j. ygyno.2017.07.130

8. Audet-Walsh E, Lepine J, Gregoire J, Plante M, Caron P, Tetu B, et al. Profiling of endogenous estrogens, their precursors, and metabolites in endometrial cancer patients: association with risk and relationship to clinical characteristics. J Clin Endocrinol Metab (2011) 96:E330-9. doi:10.1210/ jc.2010-2050

9. Dallal CM, Brinton LA, Bauer DC, Buist DS, Cauley JA, Hue TF, et al. Obesityrelated hormones and endometrial cancer among postmenopausal women: a nested case-control study within the B FIT cohort. Endocr Relat Cancer (2013) 20:151-60. doi:10.1530/ERC-12-0229

10. Brinton LA, Trabert B, Anderson GL, Falk RT, Felix AS, Fuhrman BJ, et al. Serum estrogens and estrogen metabolites and endometrial cancer risk among postmenopausal women. Cancer Epidemiol Biomarkers Prev (2016) 25:1081-9. doi:10.1158/1055-9965.EPI-16-0225

11. Dallal CM, Lacey JV Jr, Pfeiffer RM, Bauer DC, Falk RT, Buist DS, et al. Estrogen metabolism and risk of postmenopausal endometrial and ovarian cancer: the B approximately FIT Cohort. Horm Cancer (2016) 7:49-64. doi:10.1007/s12672-015-0237-y

12. Busch EL, Crous-Bou M, Prescott J, Chen MM, Downing MJ, Rosner BA, et al. Endometrial cancer risk factors, hormone receptors, and mortality prediction. Cancer Epidemiol Biomarkers Prev (2017) 26:727-35. doi:10.1158/1055-9965. EPI-16-0821

\section{ACKNOWLEDGMENTS}

We would like to thank all the study participants and those involved in the recruitment, as well as Dr. Michèle Rouleau for helpful discussions and David Simonyan of the Clinical and Evaluative Research Platform of the CHU de Québec Research Center for statistical support. The Cancer Research Society (CRS) and Canadian Institutes of Health Research (FRN-68964 to CG) supported this work. YA-D received a studentship from Fonds de Recherche du Québec - Santé (FRQS). CG holds a Tier I Canada Research Chair in Pharmacogenomics.

\section{SUPPLEMENTARY MATERIAL}

The Supplementary Material for this article can be found online at http://www.frontiersin.org/articles/10.3389/fendo.2018.00087/ full\#supplementary-material.

13. Wishart DS. Emerging applications of metabolomics in drug discovery and precision medicine. Nat Rev Drug Discov (2016) 15:473-84. doi:10.1038/ nrd.2016.32

14. Turkoglu O, Zeb A, Graham S, Szyperski T, Szender JB, Odunsi K, et al. Metabolomics of biomarker discovery in ovarian cancer: a systematic review of the current literature. Metabolomics (2016) 12:60. doi:10.1007/ s11306-016-0990-0

15. Yin R, Yang T, Su H, Ying L, Liu L, Sun C. Saturated fatty acids as possible important metabolites for epithelial ovarian cancer based on the free and esterified fatty acid profiles determined by GC-MS analysis. Cancer Biomark (2016) 17:259-69. doi:10.3233/CBM-160638

16. Zhang Y, Liu Y, Li L, Wei J, Xiong S, Zhao Z. High resolution mass spectrometry coupled with multivariate data analysis revealing plasma lipidomic alteration in ovarian cancer in Asian women. Talanta (2016) 150:88-96. doi:10.1016/j. talanta.2015.12.021

17. Bachmayr-Heyda A, Aust S, Auer K, Meier SM, Schmetterer KG, Dekan S, et al. Integrative systemic and local metabolomics with impact on survival in high-grade serous ovarian cancer. Clin Cancer Res (2017) 23:2081-92. doi:10.1158/1078-0432.CCR-16-1647

18. Xie H, Hou Y, Cheng J, Openkova MS, Xia B, Wang W, et al. Metabolic profiling and novel plasma biomarkers for predicting survival in epithelial ovarian cancer. Oncotarget (2017) 8:32134-46. doi:10.18632/oncotarget.16739

19. Trousil S, Lee P, Pinato DJ, Ellis JK, Dina R, Aboagye EO, et al. Alterations of choline phospholipid metabolism in endometrial cancer are caused by choline kinase alpha overexpression and a hyperactivated deacylation pathway. Cancer Res (2014) 74:6867-77. doi:10.1158/0008-5472.CAN-13-2409

20. Shao X, Wang K, Liu X, Gu C, Zhang P, Xie J, et al. Screening and verifying endometrial carcinoma diagnostic biomarkers based on a urine metabolomic profiling study using UPLC-Q-TOF/MS. Clin Chim Acta (2016) 463:200-6. doi:10.1016/j.cca.2016.10.027

21. Gaudet MM, Falk RT, Stevens RD, Gunter MJ, Bain JR, Pfeiffer RM, et al. Analysis of serum metabolic profiles in women with endometrial cancer and controls in a population-based case-control study. JClin Endocrinol Metab (2012) 97:3216-23. doi:10.1210/jc.2012-1490

22. Bahado-Singh RO, Lugade A, Field J, Al-Wahab Z, Han B, Mandal R, et al. Metabolomic prediction of endometrial cancer. Metabolomics (2017) 14:6. doi:10.1007/s11306-017-1290-z

23. Troisi J, Sarno L, Landolfi A, Scala G, Martinelli P, Venturella R, et al. Metabolomic signature of endometrial cancer. JProteome Res (2018) 17:804-12. doi:10.1021/acs.jproteome.7b00503

24. Shajahan-Haq AN, Boca SM, Jin L, Bhuvaneshwar K, Gusev Y, Cheema AK, et al. EGR1 regulates cellular metabolism and survival in endocrine resistan breast cancer. Oncotarget (2017) 8:96865-84. doi:10.18632/oncotarget.18292

25. Lofgren L, Stahlman M, Forsberg GB, Saarinen S, Nilsson R, Hansson GI. The BUME method: a novel automated chloroform-free 96-well total lipid extraction 
method for blood plasma. JLipid Res (2012) 53:1690-700. doi:10.1194/ jlr.D023036

26. Pearce N. Analysis of matched case-control studies. BMJ (2016) 352:1969. doi:10.1136/bmj.i969

27. Obuchowski NA. ROC analysis. AJR Am J Roentgenol (2005) 184:364-72. doi:10.2214/ajr.184.2.01840364

28. Soda K. The mechanisms by which polyamines accelerate tumor spread. J Exp Clin Cancer Res (2011) 30:95. doi:10.1186/1756-9966-30-95

29. Green ML, Chung TE, Reed KL, Modric T, Badinga L, Yang J, et al. Paracrine inducers of uterine endometrial spermidine/spermine N1-acetyltransferase gene expression during early pregnancy in the Pigl. Biol Reprod (1998) 59:1251-8. doi:10.1095/biolreprod59.5.1251

30. Pistilli MJ, Petrik JJ, Holloway AC, Crankshaw DJ. Immunohistochemical and functional studies on calcium-sensing receptors in rat uterine smooth muscle. Clin Exp Pharmacol Physiol (2012) 39:37-42. doi:10.1111/ j.1440-1681.2011.05631.x

31. Tajima M, Harada T, Ishikawa T, Iwahara Y, Kubota T. Augmentation of arginase expression in the human endometrial epithelium in the secretory phase. J Med Dent Sci (2012) 59:75-82.

32. Loftsson T, Somogyi G, Bodor N. Effect of choline esters and oleic acid on the penetration of acyclovir, estradiol, hydrocortisone, nitroglycerin, retinoic acid and trifluorothymidine across hairless mouse skin in vitro. Acta Pharm Nord (1989) 1:279-86.

33. Chughtai K, Jiang L, Greenwood TR, Glunde K, Heeren RM. Mass spectrometry images acylcarnitines, phosphatidylcholines, and sphingomyelin in MDA-MB-231 breast tumor models. J Lipid Res (2013) 54:333-44. doi:10.1194/jlr.M027961

34. Pochini L, Oppedisano F, Indiveri C. Reconstitution into liposomes and functional characterization of the carnitine transporter from renal cell plasma membrane. Biochim Biophys Acta (2004) 1661:78-86. doi:10.1016/ j.bbamem.2003.12.001

35. Schooneman MG, Vaz FM, Houten SM, Soeters MR. Acylcarnitines: reflecting or inflicting insulin resistance? Diabetes (2013) 62:1-8. doi:10.2337/ db12-0466

36. Guida M, Ligresti A, De Filippis D, D’amico A, Petrosino S, Cipriano M, et al. The levels of the endocannabinoid receptor CB2 and its ligand 2-arachidonoylglycerol are elevated in endometrial carcinoma. Endocrinology (2010) 151:921-8. doi:10.1210/en.2009-0883

37. Qin H, Ruan ZH. The role of monoacylglycerol lipase (MAGL) in the cancer progress. Cell Biochem Biophys (2014) 70:33-6. doi:10.1007/s12013-014-9899-2

38. Ahmed A, Ferriani RA, Smith SK. Activation of human endometrial phospholipase D by bradykinin. Cell Signal (1995) 7:599-609. doi:10.1016/ 0898-6568(95)00028-N

39. Ehrenfeld P, Conejeros I, Pavicic MF, Matus CE, Gonzalez CB, Quest AF, et al. Activation of kinin B1 receptor increases the release of metalloproteases- 2 and -9 from both estrogen-sensitive and -insensitive breast cancer cells. Cancer Lett (2011) 301:106-18. doi:10.1016/j.canlet.2010.09.020

40. Orchel J, Witek L, Kimsa M, Strzalka-Mrozik B, Kimsa M, Olejek A, et al. Expression patterns of kinin-dependent genes in endometrial cancer. Int J Gynecol Cancer (2012) 22:937-44. doi:10.1097/IGC.0b013e318259d8da

41. Naitoh K, Honjo H, Yamamoto T, Urabe M, Ogino Y, Yasumura T, et al. Estrone sulfate and sulfatase activity in human breast cancer and endometrial cancer. J Steroid Biochem (1989) 33:1049-54. doi:10.1016/0022-4731(89)90408-1

42. Lepine J, Audet-Walsh E, Gregoire J, Tetu B, Plante M, Menard V, et al. Circulating estrogens in endometrial cancer cases and their relationship with tissular expression of key estrogen biosynthesis and metabolic pathways. J Clin Endocrinol Metab (2010) 95:2689-98. doi:10.1210/jc.2010-2648

43. Audet-Delage Y, Gregoire J, Caron P, Turcotte V, Plante M, Ayotte P, et al. Estradiol metabolites as biomarkers of endometrial cancer prognosis after surgery. JSteroid Biochem Mol Biol (2018) 178:45-54. doi:10.1016/ j.jsbmb.2017.10.021

44. Sinreih M, Knific T, Anko M, Hevir N, Vouk K, Jerin A, et al. The significance of the sulfatase pathway for local estrogen formation in endometrial cancer. Front Pharmacol (2017) 8:368. doi:10.3389/fphar.2017.00368

45. di Salvo ML, Contestabile R, Paiardini A, Maras B. Glycine consumption and mitochondrial serine hydroxymethyltransferase in cancer cells: the heme connection. Med Hypotheses (2013) 80:633-6. doi:10.1016/j.mehy. 2013.02.008
46. Genkinger JM, Friberg E, Goldbohm RA, Wolk A. Long-term dietary heme iron and red meat intake in relation to endometrial cancer risk. Am J Clin Nutr (2012) 96:848-54. doi:10.3945/ajcn.112.039537

47. Decollogne S, Joshi S, Chung SA, Luk PP, Yeo RX, Nixdorf S, et al. Alterations in the mitochondrial responses to PENAO as a mechanism of resistance in ovarian cancer cells. Gynecol Oncol (2015) 138:363-71. doi:10.1016/ j.ygyno.2015.06.018

48. Tran B, Hamilton AL, Horvath L, Lam M, Savas PS, Grimison PS, et al. First-in-man trial of 4 -(N-(S-penicillaminylacetyl)amino) phenylarsonous acid (PENAO) as a continuous intravenous infusion (CIVI), in patients (pt) with advanced solid tumours. JClin Oncol (2016) 34:e14025. doi:10.1200/ JCO.2016.34.15_suppl.e14025

49. Rizner TL, Penning TM. Role of aldo-keto reductase family 1 (AKR1) enzymes in human steroid metabolism. Steroids (2014) 79:49-63. doi:10.1016/ j.steroids.2013.10.012

50. Guillemette C, Levesque E, Rouleau M. Pharmacogenomics of human uridine diphospho-glucuronosyltransferases and clinical implications. Clin Pharmacol Ther (2014) 96:324-39. doi:10.1038/clpt.2014.126

51. Kurogi K, Liu TA, Sakakibara Y, Suiko M, Liu MC. The use of zebrafish as a model system for investigating the role of the SULTs in the metabolism of endogenous compounds and xenobiotics. Drug Metab Rev (2013) 45:431-40. doi:10.3109/03602532.2013.835629

52. Jiang M, Xu M, Ren S, Selcer KW, Xie W. Transgenic overexpression of steroid sulfatase alleviates cholestasis. Liver Res (2017) 1:63-9. doi:10.1016/ j.livres.2017.03.001

53. Germain AM, Kato S, Carvajal JA, Valenzuela GJ, Valdes GL, Glasinovic JC. Bile acids increase response and expression of human myometrial oxytocin receptor. Am J Obstet Gynecol (2003) 189:577-82. doi:10.1067/ S0002-9378(03)00545-3

54. Li M, Cai SY, Boyer JL. Mechanisms of bile acid mediated inflammation in the liver. Mol Aspects Med (2017) 56:45-53. doi:10.1016/j.mam.2017.06.001

55. Zhuang S, Li Q, Cai L, Wang C, Lei X. Chemoproteomic profiling of bile acid interacting proteins. ACS Cent Sci (2017) 3:501-9. doi:10.1021/ acscentsci.7b00134

56. Ghezzi F, Cromi A, Siesto G, Giudici S, Serati M, Formenti G, et al. Prognostic significance of preoperative plasma fibrinogen in endometrial cancer. Gynecol Oncol (2010) 119:309-13. doi:10.1016/j.ygyno.2010.07.014

57. Seebacher V, Polterauer S, Grimm C, Husslein H, Leipold H, HeflerFrischmuth K, et al. The prognostic value of plasma fibrinogen levels in patients with endometrial cancer: a multi-centre trial. Br J Cancer (2010) 102:952-6. doi:10.1038/sj.bjc.6605547

58. Suh DH, Kim HS, Chung HH, Kim JW, Park NH, Song YS, et al. Pre-operative systemic inflammatory response markers in predicting lymph node metastasis in endometrioid endometrial adenocarcinoma. Eur J Obstet Gynecol Reprod Biol (2012) 162:206-10. doi:10.1016/j.ejogrb.2012.02.028

59. Uccella S, Cromi A, Vigetti D, Cimetti L, Deleonibus S, Casarin J, et al. Endometrial cancer cells can express fibrinogen: Immunohistochemistry and RT-PCR analysis. J Obstet Gynaecol (2016) 36:353-8. doi:10.3109/01443615. 2015.1065231

60. Zhou X, Wang H, Wang X. Preoperative CA125 and fibrinogen in patients with endometrial cancer: a risk model for predicting lymphovascular space invasion. J Gynecol Oncol (2017) 28:e11. doi:10.3802/jgo.2017.28.e11

61. Nozawa S, Narisawa S, Kojima K, Sakayori M, Iizuka R, Mochizuki H, et al. Human monoclonal antibody (HMST-1) against lacto-series type 1 chain and expression of the chain in uterine endometrial cancers. Cancer Res (1989) 49:6401-6.

62. Knapp P, Baranowski M, Knapp M, Zabielski P, Blachnio-Zabielska AU, Gorski J. Altered sphingolipid metabolism in human endometrial cancer. Prostaglandins Other Lipid Mediat (2010) 92:62-6. doi:10.1016/j.prostaglandins. 2010.03.002

63. Mojakgomo R, Mbita Z, Dlamini Z. Linking the ceramide synthases (CerSs) 4 and 5 with apoptosis, endometrial and colon cancers. Exp Mol Pathol (2015) 98:585-92. doi:10.1016/j.yexmp.2015.03.019

64. Tajima T, Miyazawa M, Hayashi M, Asai S, Ikeda M, Shida M, et al. Enhanced expression of hydroxylated ceramide in well-differentiated endometrial adenocarcinoma. Oncol Lett (2017) 13:45-50. doi:10.3892/ol.2016.5410

65. Tanaka K, Mikami M, Aoki D, Kiguchi K, Ishiwata I, Iwamori M. Expression of sulfatide and sulfated lactosylceramide among histological types of 
human ovarian carcinomas. Hum Cell (2015) 28:37-43. doi:10.1007/ s13577-014-0100-4

66. Merritt MA, Cramer DW. Molecular pathogenesis of endometrial and ovarian cancer. Cancer Biomark (2010) 9:287-305. doi:10.3233/CBM-20110167

67. Cramer DW. The epidemiology of endometrial and ovarian cancer. Hematol Oncol Clin North Am (2012) 26:1-12. doi:10.1016/j.hoc.2011.10.009

68. Knapp P, Bodnar L, Blachnio-Zabielska A, Swiderska M, Chabowski A. Plasma and ovarian tissue sphingolipids profiling in patients with advanced ovarian cancer. Gynecol Oncol (2017) 147:139-44. doi:10.1016/j.ygyno.2017. 07.143
Conflict of Interest Statement: The authors declare that there are no actual or potential conflicts of interest that could inappropriately influence, or be perceived to influence, this work.

Copyright (c) 2018 Audet-Delage, Villeneuve, Grégoire, Plante and Guillemette. This is an open-access article distributed under the terms of the Creative Commons Attribution License (CC BY). The use, distribution or reproduction in other forums is permitted, provided the original author(s) and the copyright owner are credited and that the original publication in this journal is cited, in accordance with accepted academic practice. No use, distribution or reproduction is permitted which does not comply with these terms. 